\title{
Educación ambiental: itinerario en la naturaleza y su relación con conectividad, preocupaciones ambientales y conducta
}

\section{Environmental education: itineraries in nature and their relationship with connectedness, environmental concerns and behavior}

Recibido: 15/07/2013

Revisado: 23/08/2013

Aceptado: 22/10/2013
Pablo Olivos-Jara,

Universidad de Castilla - La Mancha, Espańa

Juan-Ignacio Aragonés y

Universidad Complutense de Madrid, España

Oscar Navarro-Carrascal

Universidad de Nantes, Francia

Contacto con los autores: Pablo Olivos Jara. Facultad de Relaciones Laborales y Recursos Humanos, Universidad de Castilla-La Mancha. Plaza de la Universidad 1, CP. 02071, Albacete, España. Email: pablo.olivos@ uclm.es; Tlf: +34967599200, Ext. 2174.

Agradecimientos: Al CENEAM, por su apoyo técnico y logístico.

\section{Resumen}

Albeit there is research about basic concepts involved in environmental education, enough evidence is lacking about the experiences of contact with natural environments, so common in this field, and even less about his relationship with Connectedness with Nature, Environmental Concerns and Pro-environmental Behavior. We report results of two studies; the first involving 286 psychology students who completed a questionnaire measuring Connectedness, Concerns, Behavior and Socio-demographic and Sociocultural variables. In the second study, 40 students participated in an excursion across a natural environment, completing pre and post measures of Connectedness. The main results show that the concerns are related to demographic and socio-cultural variables such as gender, political ideology and religiosity, but not to Connectedness with Nature, which only increases after contact with a
Las excursiones e itinerarios a través de entornos naturales son frecuentes en la educación ambiental, pero existe poca evidencia empírica de investigaciones que aporten resultados que permitan comprender mejor los efectos de las experiencias de contacto directo con ambientes naturales, y menos aún acerca de su relación con la conectividad con la naturaleza, las preocupaciones ambientales y el comportamiento proambiental. Se llevan a cabo dos estudios, en el primero participan 286 estudiantes de psicología, quienes completan un cuestionario que mide conectividad, preocupación ambiental, conducta y variables sociodemográficas y socioculturales. En el segundo estudio, 40 alumnos participan en una excursión por un ambiente natural, completando medidas pre y post de conectividad. Los resultados indican que la preocupación ambiental está relacionada con variables sociodemográficas y socioculturales 
natural environment. We discuss the implications of the findings for environmental education.

Key words: connectedness with nature, environmental identity, environmental education, egobiocentrism, contact with nature

Tal vez el campo de la educación ambiental sea uno de los más prolíficos en cuanto a contenidos y didáctica, pero también es uno en los que persisten lagunas teóricoconceptuales que acompañan la planificación del proceso de enseñanza-aprendizaje (Nielsen et al., 2012). Las políticas de educación ambiental, o de educación para el desarrollo sostenible, suelen carecer de un análisis conceptual profundo y explícito del fin, los medios, la visión de ser humano y la relación que estas ideas guardan con el ambiente, a pesar de que subyacen como supuestos implícitos entre los actores encargados de diseñarlas y ejecutarlas (GonzálezGaudiano, 2005; Hesselink, van Kempen \& Wals, 2000; Navarro, 2011; Olivos \& González, 2005). Además, con frecuencia se observan actividades de educación ambiental que mezclan azarosamente objetivos para la formación de valores, el aprendizaje de contenidos, y la promoción de comportamientos ecológicos, sostenibles y/o proambientales, indistintamente y sin un análisis crítico de los mismos (O’Donoghue, 2006; Olivos, 2006; Olivos $\&$ Moyano, 2004).

Thomashow (1995) propuso que una educación ambiental implica un sentido de identidad ecológica, cuyo propósito de fondo es producir un cambio personal a partir de un punto de vista ecológico, en el sentido biológico de la expresión. A diferencia de las hipótesis socioculturales, según este autor el espacio natural ofrece un lenguaje, un contexto, un ancla moral y el aprendizaje reflexivo que conecta las elecciones vitales de una persona con su visión ecológica del mundo, sirviendo como guía para coordinar significados, una transición hacia una nueva forma de ser uno mismo en el mundo. Desde esta perspectiva, un educador ambiental debe conducir a través de los escenarios de aprendizaje para que las personas puedan verse reflejadas en las posibles identificaciones con la naturaleza y sus propiedades. como el género, la ideología política y la religiosidad; no así la conectividad con la naturaleza, que solo aumenta tras el contacto con un ambiente natural. Se discuten los alcances de los resultados del estudio para la educación ambiental.

Palabras clave: conectividad con la naturaleza, identidad ambiental, educación ambiental, egobiocentrismo, contacto con la naturaleza

En un sentido similar se ha propuesto el concepto de Conectividad con la Naturaleza (Mayer \& Franz, 2004), que pretende reflejar una sensación de parentesco y una experiencia individual afectiva de unión con la naturaleza de carácter implícito (Schultz, Shriver, Tabanico, \& Khazian, 2004). Aun cuando se ha cuestionado el carácter emocional e implícito de las medidas de conectividad (Brügger, Kaiser, \& Roczen, 2011; Olivos \& Aragonés, 2013a; Perrin \& Benassi, 2009), estudios han mostrado cómo la construcción de identidades supraordenadas o metapersonales guardan una estrecha relación con la conectividad con la naturaleza y el comportamiento proambiental (Arnocky, Stroink, \& DeCicco, 2007; Hoot \& Friedman, 2011; Olivos \& Aragonés, 2013b).

Las experiencias de contacto directo con ambientes naturales son frecuentes en la educación ambiental. Por una parte, existen evidencias en favor del diseño de talleres de capacitación en entornos naturales, al aire libre, para el desarrollo de la personalidad de adolescentes y jóvenes (Fangler \& Schwarzer, 2008; Martens \& Bauer, 2008), donde las actividades han sido diseñadas para transmitir valores, generar hábitos y crear un sentido de responsabilidad compartida. También hay estudios que han investigado algunos efectos significativos de actividades de educación ambiental sobre las conductas proambientales. Sin embargo, este no es el caso cuando se abordan el conocimiento y los afectos positivos hacia el medio ambiente (Erdoğan, 2011).

Sin embargo, faltan estudios acerca de los efectos de las actividades de educación ambiental, realizadas en contacto con la naturaleza, sobre la conectividad y el significado subjetivo de esas experiencias. Algunos estudios recientes han mostrado el efecto que pueden tener los cursos de educación ambiental en favor del aumento de la conectividad con la 
naturaleza (Ernst \& Theimer, 2011; Liefländer, Fröhlich, Bogner \& Schultz, 2013). Algunos de estos resultados han sido más duraderos en grupos de niños (9-11 años), tras medir la conectividad cuatro semanas después de terminado un curso de cuatro días de duración. Sin embargo, como los propios autores señalan, estos estudios suelen carecer de medidas de línea base fiables, y no evalúan específicamente la participación en actividades de contacto directo con la naturaleza.

Por otra parte, sí que existe gran cantidad de evidencia sobre los efectos positivos que el contacto con ambientes naturales tiene en la salud y el bienestar de las personas (e.g. R. Kaplan, 2001; S. Kaplan, 1995; Staats, Gatersleben $\&$ Hartig, 1997). También se ha observado un aumento en los niveles de conectividad con la naturaleza tras participar en actividades ambientales consistentes en estancias o recorridos de diferente duración por un área natural (Mayer, Frantz, Bruehlman-Senecal, \& Dolliver, 2009; Schultz \& Tabanico, 2007), incluso comparándolo con experiencias en ambientes urbanos, reales o virtuales. Además se ha observado que la conectividad tiene un efecto mediador en el aumento de los estados de ánimo positivos derivados de estas experiencias. Sin embargo, hasta la fecha siguen siendo escasas las medidas longitudinales de los impactos de estas actividades en el área de la educación ambiental.

Otro aspecto relevante de las experiencias de contacto con la naturaleza consiste en los significados que se les atribuyen o las experiencias subjetivas que evocan. En una serie longitudinal de estudios cualitativos, Schroeder (1991, 2002, 2007) pidió a personas con especial atracción por espacios al aire libre que describieran un lugar preferido, y posteriormente que escribieran qué significaba ese lugar para ellos, señalando sus pensamientos, sensaciones, recuerdos, experiencias, y todo aquello que les viniera a la mente en relación con ese lugar. El autor observó algunos temas recurrentes en todas las descripciones, tales como belleza, tranquilidad, naturalidad, lejanía, refugio y escape, emoción, admiración, lazos sociales, historia familiar, y patrimonio. Uno de los hallazgos más interesantes para los propósitos de la presente investigación fue que en algunos casos la experiencia personal con el lugar o ambiente les otorgaba a los participantes una sensación de su propia conexión con la naturaleza, así como de sentirse incluidos en el orden natural del entorno. Este contenido, según observó el autor, coexistía con una paradójica sensación de que la civilización humana está separada y reñida con la naturaleza, y una sensación personal de necesitar estar lejos de otras personas y de la civilización. Schroeder interpretó estos resultados como una compleja relación gestáltica de la relación ser humano-ambiente natural.

A propósito de la planificación de contenidos y actividades en educación ambiental, y teniendo en cuenta los antecedentes revisados, en la presente investigación se estudia la experiencia de contacto directo con entornos naturales, particularmente su relación con la conectividad con la naturaleza, la constelación de creencias subyacentes a las preocupaciones ambientales, y la intención de comportamientos proambientales, así como la descripción de los significados atribuidos a estas experiencias. La investigación se lleva cabo mediante dos estudios que cubren los tres objetivos siguientes:

En primer lugar se busca describir las relaciones entre conectividad con la naturaleza, preocupación ambiental y comportamientos proambientales. $\mathrm{Al}$ respecto, se espera que la conectividad tenga una correlación fuerte y positiva con preocupaciones egobiocéntricas y conductas proambientales, en coherencia con resultados previos (Olivos \& Aragonés, 2011; Olivos, Aragonés \& Amérigo, 2011).

El segundo objetivo consiste en describir los efectos de una experiencia de contacto con un entorno sobre la conectividad con la naturaleza. De acuerdo con los resultados presentados en otros estudios (e.g. Mayer et al., 2009; Schultz \& Tabanico, 2007), se espera que la conectividad aumente después de una experiencia de este tipo.

El tercer objetivo busca describir la experiencia subjetiva que evoca una actividad de contacto con un ambiente natural. Se espera encontrar una organización conceptual similar a la encontrada en estudios previos (Schroeder, 2007; Thomashow, 1995), referida a la forma en que los participantes experimentan este ambiente.

Para llevar a cabo esto se analizan los resultados de dos estudios que se describen a continuación. El primero se hace cargo del primer objetivo, y el segundo estudio aborda los dos siguientes. 


\section{Estudio 1}

\section{Método}

Participantes. En este estudio participaron 286 estudiantes de psicología de la Universidad Complutense de Madrid. La edad media de la muestra fue de 21.4 años $(D T=3.78)$, y está compuesta por $20 \%$ varones y $80 \%$ mujeres.

Instrumentos y procedimiento. El instrumento que se utilizó fue un cuestionario autoadministrado que, además de preguntas sociodemográficas (edad, género, grado de religiosidad e ideología política), contenía las siguientes escalas:

Conectividad con la Naturaleza (CNS) de Mayer y Frantz (2004), compuesta por 14 ítems con respuestas tipo Likert de 5 puntos ( $1=$ muy en desacuerdo; $5=$ muy de acuerdo), en la versión de Olivos, Aragonés y Amérigo (2011).

Inclusión del Ambiente en el Self, en la versión de Olivos y Aragonés (2013b), es otra forma de medir conectividad en una escala de 14 ítems que utilizan diagramas de Venn con puntuación tipo likert de cinco puntos (1=baja conectividad; $5=$ alta conectividad). Está compuesta por dos subescalas: Inclusión del Ambiente Construido (IACS) y del Ambiente Natural (IANS) en el Self.

Con el fin de medir la preocupación ambiental se recurre a las dimensiones Antropocentrismo (ANT), Egobiocetrismo (EGO) y Biosferismo (BIO) utilizadas por Amérigo et al. (2007). Cada una de ellas está compuesta por 5 ítems tipo Likert de 5 puntos ( $1=$ muy en desacuerdo; $5=$ muy de acuerdo).

Autoinforme de Comportamientos Proambientales (CPA, de Sevillano, 2007), compuesta por 11 ítems tipo Likert de 5 puntos ( $1=$ nunca; $5=$ siempre).

Cada aplicación tardó aproximadamente 20 minutos, y se realizó en las aulas universitarias, al comenzar una clase.

\section{Resultados}

Según las puntuaciones observadas en la escala CNS $(\alpha=.82)$, los participantes tienen una media de conectividad de 3.52 $(D T=0.52)$, lo que indica que están relativamente conectados con la naturaleza.
Las escalas IANS e IACS también alcanzan una fiabilidad alta (IANS $\alpha=.88$; IACS $\alpha=.80$ ). Una prueba $t$ para muestras relacionadas $(t=10.62 ; \mathrm{gl}=285 ; p<.01)$ indica que hay diferencias significativas entre ellas, por lo que los participantes están más conectados con el ambiente natural (IANS $M=3.36, D T=0.77$ ) que con el ambiente construido (IACS $M=2.64, D T=0.78$ ).

Las escalas Egobiocéntrica $(\alpha=.78)$ y Antropocéntrica $(\alpha=.76)$ logran una fiabilidad aceptable. En cambio, la escala Biosférica muestra una fiabilidad más baja $(\alpha=.55)$, pero esto no impide su utilización, como se ha argumentado en estudios anteriores (Olivos y Aragonés, 2013a). Se observa también una puntuación media ligeramente mayor en Biosferismo $(M=4.14, D T=0.47)$ que en Egobiocentrismo $(M=3.97, D T=.57)$, y a su vez ambas tienen una puntuación mucho mayor que Antropocentismo $(M=2.17, D T=0.63)$.

La escala CPA tiene una fiabilidad moderada $(\alpha=.69)$. La puntuación media obtenida por los participantes $(M=3.53$ y $D T=0.56)$ indica que con relativa frecuencia realizan conductas proambientales.

En cuanto a la relación entre Conectividad, Preocupación Ambiental y Comportamiento, se correlacionaron los resultados de las escalas de Conectividad (CNS, IANS, IACS), Preocupaciones ambientales (EGO, BIO, ANT), y la CPA.

\begin{tabular}{|c|c|c|c|}
\hline & CNC & IANS & $I A C S$ \\
\hline Egobiocentrismo & $.557^{\star \star}$ & $.476^{\star \star}$ & $-.275^{\star \star}$ \\
\hline Biosferismo & $.410^{* *}$ & $.396^{* *}$ & $-.245^{\star \star}$ \\
\hline Antropocentrismo & $-.125^{\star}$ & $-.198^{\star *}$ & $.162^{* *}$ \\
\hline CPA & $.303^{\star \star}$ & $.243^{\star \star}$ & $-.258^{\star \star}$ \\
\hline
\end{tabular}

Se observa que las medidas de Conectividad con la Naturaleza (CNS e IANS) correlacionan positivamente con las creencias ambientales EGO y BIO, y negativamente con ANT, siendo estas las correlaciones más altas que muestra la tabla. Según lo esperado, el resultado inverso se observa entre 
la escala IACS y las Preocupaciones Ambientales. Respecto del $\mathrm{CPA}$, todas las correlaciones son significativas, aunque débiles. Las correlaciones entre CPA y CNS e IANS son positivas según lo esperado, mientras que es negativa con la IACS.

En las medidas de Conectividad (CNS, IANS, IACS) no se observan diferencias significativas según el género, ni correlaciones significativas según la edad. Tampoco se observan diferencias significativas según religiosidad, ni ideología política.

En el CPA tampoco se observan diferencias significativas según género, ideología política ni religiosidad.

En Preocupación Ambiental tampoco se observan diferencias según género en ninguna de las tres dimensiones empleadas, pero sí se observan diferencias según religiosidad $\left(F_{[2 ; 277]}=3.87 ; p<.05\right)$. Los participantes con creencias "religiosas intermedias" $(M=2.26, D T=0.615)$ son más Antropocéntricos que los participantes ateos $(M=2.03, D T=0.617)$, aunque ambos grupos muestran una preocupación moderada en esta dimensión. También se observan diferencias según ideología política, en las creencias Biosféricas $(t=-2.93 ; \mathrm{gl}=275 ; p<.01)$ y Antropocéntricas $(t=4.51 ; \mathrm{gl}=174.93 ; p<.001)$. Los participantes cuya orientación política es de izquierda $(M=4.20 ; D T=0.463)$ son más Biosféricos que los de derecha $(M=4.03, D T=0.455)$, y éstos últimos son más Antropocéntricos $(M=2.42, D T=0.626)$ que los de izquierda $(M=2.06, D T=0.600)$.

\section{Estudio 2}

Como se ha comentado en la introducción, se han llevado a cabo estudios que han comprobado el aumento en los niveles de Conectividad con la Naturaleza después del contacto directo con ambientes naturales. Schultz y Tabanico (2007) midieron estos cambios utilizando una medida implícita, el IAT-Nature, después de que un grupo de personas pasaran entre cuatro horas y un día dentro de un parque natural. No obstante, otras experiencias han demostrado que la conectividad medida con la CNS también aumenta, incluso después de un paseo de diez minutos (Mayer et al., 2009). Sin embargo estos estudios han comprobado que el efecto señalado no se produce tras visitar áreas verdes urbanas, ambientes construidos en general, o después de realizar sólo actividades de entretenimiento. A pesar de estos hallazgos, se estima que son escasos los estudios que permiten indagar en el contenido evocado por estas actividades, y que subyacen a las experiencias de contacto con la naturaleza.

\section{Método}

Participantes. En este estudio participaron 40 estudiantes que habían participado antes en el estudio 1 . Su promedio de edad es de 25 años $(D T=4.83)$ y la distribución por género fue de $82.5 \%$ mujeres y $17.5 \%$ varones.

Instrumentos y procedimiento. El instrumento utilizado fue un cuestionario autoadministrado, similar al empleado en el estudio anterior, que solamente estaba compuesto por la escala de Conectividad con la Naturaleza (CNS), además de algunas preguntas de interés general sobre la experiencia previa de los participantes en actividades similares en el mismo entorno.

Por otra parte y siguiendo los estudios de Schroeder (2007), los participantes también tuvieron que describir brevemente por escrito el lugar visitado y lo que significó para ellos. Estos resultados se analizaron aplicando una técnica de análisis lexicográfico - el Open Coding (e.g. Strauss \& Corbin, 1994) - para seguir el mismo procedimiento de análisis cualitativo llevado a cabo por Schroeder. De este modo, se confeccionaron categorías asociando los principales contenidos extraídos de las unidades de análisis (significantes), y una vez confeccionadas las categorías se realizó un conteo de la frecuencia de menciones y un cálculo de la proporción de cada categoría (expresada en porcentajes) en relación con el total de nominaciones en cada pregunta, para conocer las más nombradas. Los resultados se representan en figuras utilizando la aplicación Wordle, para la confección de nubes de palabras según las frecuencias de las unidades significantes.

La cumplimentación de los instrumentos se hacía después de que los participantes hicieran un itinerario guiado por un educador ambiental del Centro Nacional de Educación Ambiental (CENEAM) 25 días después de haber participado en el estudio 1. La duración del recorrido era de aproximadamente dos horas a través de una ruta predefinida de bosque de montańa ubicada en la Sierra de Guadarrama. A lo largo de la ruta, los participantes iban observando elementos emblemáticos del paisaje de la sierra, oían explicaciones acerca del ecosistema de la zona y vivenciaban el entorno. Tanto el diseño de la ruta, como el guión de la actividad educativa se desarrolló con al apoyo de los profesionales del CENEAM. 
Del total de los participantes, solamente tres ya habían visitado el lugar, y de ellos el que lo había hecho más recientemente había sido hacia diez años, por lo que se descartan los efectos derivados del conocimiento previo del lugar.

\section{Resultados}

La puntuación media de los participantes en la escala CNS antes de la excursión $(M=3.54, D T=0.51)$ indica que estaban relativamente conectados con la naturaleza. Esto fue igual para el grupo de estudiantes del estudio 1 que no participaron en esta experiencia $(M=3.51$, $D T=0.52)$, de hecho, no hay diferencias significativas entre las puntuaciones de Conectividad entre ambos grupos de alumnos $(t=0.38 ; g l=270 ; p=.704)$.

Para observar en qué medida la conectividad de los participantes había variado tras la realización de la experiencia se llevó a cabo una prueba $t$ para muestras relacionadas entre las puntuaciones que habían dado en el momento de realización del estudio 1 y las dadas nada más finalizar el itinerario. El resultado indica que hay diferencias significativas en la CNS $(t=-2.07 ; g l=36 ; p<.05)$ antes $(M=3.54, D T=0.523)$ y después $(M=3.70, D T=0.515)$ de la excursión.

El resultado indica que aumenta el grado de conexión con la naturaleza, en línea con las investigaciones precedentes, después del contacto directo con un entorno natural en una actividad de educación ambiental.

El análisis de las respuestas a las preguntas abiertas, siguiendo el procedimiento de Schroeder (2007), muestra algunas ideas fuerza sobre lo que fue la experiencia para los sujetos. En primer lugar (figura 1), las respuestas a la pregunta en la que los participantes tenían que describir brevemente el lugar que acaban de visitar, muestra cómo elementos más destacados del paisaje para ellos: la flora (25.67\% de las nominaciones), caracterizada principalmente por el bosque, los árboles en general y algunos específicos como el Roble y el Pino; los sonidos de la naturaleza (9.58\%), principalmente de los pájaros y luego los del agua; la fauna ( $8.81 \%)$, siendo los pájaros en general lo más mencionado; y en cuarto lugar un conjunto de respuestas referidas a aspectos del paisaje que trascienden lo físico, más cercano a la experiencia de los participantes, es decir, lo pacífico y tranquilo del lugar (6.51\%).

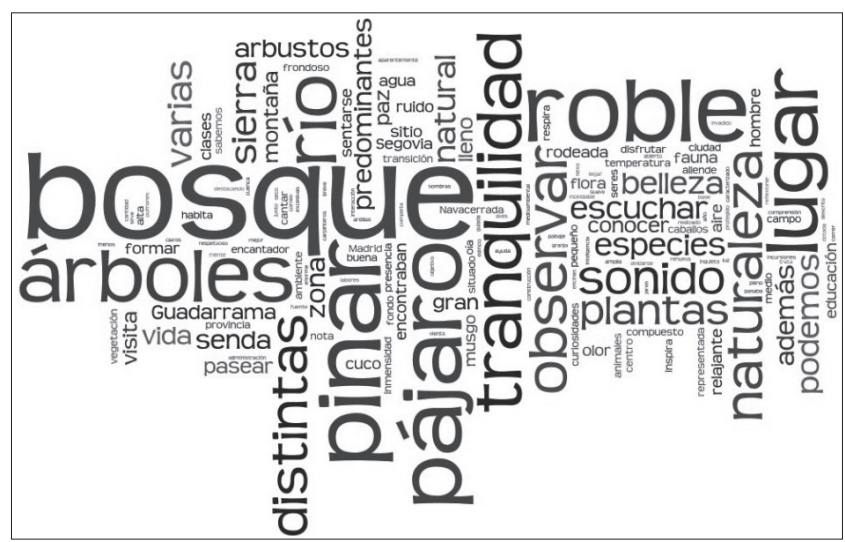

Figura 1.- Nube de palabras (unidades significantes) según frecuencia de nominaciones para la descripción del lugar visitado.

En segundo lugar, cuando los participantes tuvieron que indicar lo que el lugar visitado significó para ellos (figura 2), los contenidos más relevantes tuvieron que ver con recuerdos $(23.78 \%)$, siendo los recuerdos de infancia los más nombrados, seguidos de los recuerdos de las excursiones del colegio y de algún lugar en particular. Algunos ejemplos representativos de estas expresiones son las siguientes:

"Pues me ha recordado que iba en mi infancia a mi pueblo que estaba rodeado de montańas y cuando trabajaba como monitora en granjas escuelas...”. (Mujer, 26 años)

"Me he acordado de la excursión que hice en el año 97 con el instituto. La primera vez que vi el muérdago en mi vida fue aquí y lo he recordado al volver. Me han venido muchos recuerdos de esa época de mi vida y me parece mentira que ya hayan pasado 10 años...". (Mujer, 26 años)

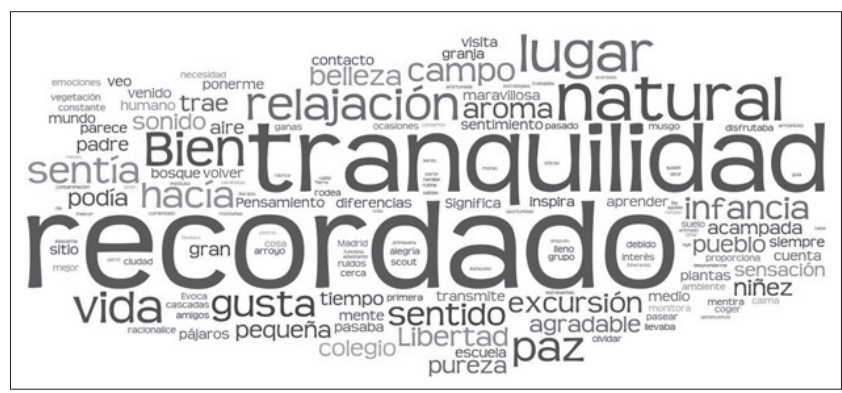

Figura 2.- Nube de palabras (significantes) según frecuencia de nominaciones para la descripción de lo que el lugar visitado significó para los participantes.

Otros contenidos frecuentemente mencionados cuando los participantes mencionaron lo que el lugar visitado significó para ellos dicen relación con: la paz y tranquilidad del lugar (25\%), que también incluye la sensación de relajación; la sensación de bienestar (9.76\%); la belleza y grandiosidad de la naturaleza (6.71\%); entre otros. 
Además del análisis de contenido y frecuencias, las respuestas también se analizaron desde el punto de vista de la experiencia emocional del ambiente. Utilizando las mismas unidades de análisis obtenidas a partir del Open Coding, y como categorías de clasificación los elementos connotativos que caracterizan la evaluación del paisaje (e.g. Corraliza, 2000), se observan respuestas relativas a los cuatro componentes emocionales (agrado, activación, impacto y control), predominando entre ellos el Agrado o sensación de bienestar provocado por la visita (tabla 2).

\begin{tabular}{|c|c|}
\hline Categoría de evaluación & Respuestas \\
\hline Agrado & $\begin{array}{l}\text { "Bonito, cómodo y sobre todo muy tranquilo, } \\
\text { relajante" }\end{array}$ \\
\hline Activación & $\begin{array}{l}\text { "La emociones son de gran alegría, } \\
\text { sentimiento de vitalidad y con ganas de } \\
\text { movimiento y tranquilidad" }\end{array}$ \\
\hline Impacto & $\begin{array}{l}\text { "Pequeño paraíso lleno de vida por todas } \\
\text { partes, con un aroma inolvidable que renueva } \\
\text { los pulmones..." }\end{array}$ \\
\hline Control & $\begin{array}{l}\text { "Es un lugar con mucha luz en la que se } \\
\text { perciben mejor los colores y los olores cuyo } \\
\text { ruido de fondo es el sonido de los pájaros } \\
\text { y cuya inmensidad está representada por } \\
\text { inmensos pinos y robles" }\end{array}$ \\
\hline
\end{tabular}

Hay otro grupo de resultados pertinentes para el análisis que se lleva a cabo en la presente investigación en la línea de los antecedentes acerca de la sensación de conexión individual con la naturaleza a partir de la experiencia personal con el lugar. En este estudio aparece una serie de contenidos referidos a la sensación de estar incluidos en la naturaleza desde un punto de vista individual, alejado de otras personas y de la ciudad, que se conciben separados o contrarios a la naturaleza. Algunos ejemplos serían los siguientes:

\footnotetext{
"Silencio, tranquilidad, vida, alejado del ser humano. Me ha recordado a una visita que realicé a Rascafría hace dos meses. Ajeno y tranquilo". (Varón, 27 años)

"Me hace desconectar, sentirme a gusto, desprenderme de muchas de las cosas que hay en la ciudad y que no me gustan nada. Me hace recordar mi infancia, muy feliz, cuando estoy en estos sitios, cada vez me convenzo más de que tengo que vivir cerca”. (Mujer, 26 años)

"Es un lugar de liberación, en el cual te pones en pleno contacto con la naturaleza y el medio ambiente. Me transmite tranquilidad y desahogo del mundo urbano". (Mujer, 23 años)
}

Estas respuestas muestran cómo los participantes son conscientes de una sensación de relajación y tranquilidad, debido a un doble efecto; por una parte la experiencia de contacto directo con las propiedades físicas más salientes del paisaje, y por otra la ausencia de otros estímulos que sobrecargan la experiencia de los participantes en la ciudad.

\section{Discusión}

La CNS logra una buena consistencia interna, mostrando que los participantes están relativamente conectados con la naturaleza. Al mismo tiempo se confirma la fortaleza de las subescalas IANS e IACS, siendo la primera una medida alternativa de conectividad con la naturaleza. Las puntuaciones de ambas escalas muestran en este caso que los participantes están más conectados con el ambiente natural que con el ambiente construido.

La CNS correlaciona positivamente con la escala de comportamiento proambiental, y ambas escalas de conectividad con la naturaleza (CNS e IANS) correlacionan positivamente con las creencias egobiocéntricas y biosféricas, y negativamente con el antropocentrismo, como también se esperaba. Su relación más fuerte con el egobiocentrismo va en el sentido de lo planteado, relativo a un componente hedónico en la valoración de la naturaleza. Desde el punto de vista de la evaluación del ambiente natural, y a partir de resultados que vinculan conectividad y la preocupación ambiental egobiocéntrica (Mayer et al., 2009; Olivos, Aragonés \& Amérigo, 2011), el agrado coincidiría con una valoración asociada a la sensación de bienestar con la naturaleza. Esto podría estar relacionado con los estudios sobre tono hedónico y propiedades ambientales en la predicción de preferencias por ambientes naturales (e.g. Hagerhall, 2001; Han, 2007; R. Kaplan, 2001; S. Kaplan, 1995).

Este resultado es relevante, ya que mientras el biosferismo pone la preocupación ambiental fuera del sujeto, centrada en el valor intrínseco del medio ambiente, el egobiocentrismo mantiene esa preocupación pero valorando el medio ambiente a través de una relación donde el sujeto es beneficiario directo del contacto directo con la naturaleza. Pero no es un beneficio pragmático, como se desprende del Antropocentrismo, sino subjetivo o trascendental. En este sentido, la conectividad con la naturaleza que se deriva de un tipo de identidad supraordenada (Olivos \& Aragonés, 2013b) no significa la disolución del self, donde todo lo 
que importa es la naturaleza, sino más bien corresponde a un espacio de interacción donde el self y la naturaleza se asocian en una idea de unidad.

En este sentido, la relación que muestran variables como la ideología política o la religiosidad con las creencias ambientales subyacentes a la preocupación ambiental sugieren que los jóvenes más conservadores (de religiosidad moderada y orientados políticamente hacia la derecha) son proclives a creencias antropocéntricas, a diferencia de los más progresistas (ateos y orientados políticamente a la izquierda) que son más biocéntricos. De hecho, es necesario un análisis más detenido del comportamiento proambiental en relación a este tipo de variables sociodemográficas y socioculturales, pues según el estudio de Olivos y Talayero (2013) subyacen a esta escala tres dimensiones: Ecologista (ECO, que incluyen conductas de voluntariado ambiental y elecciones de consumo ecológico, entre otras), Urbanista (URB, que incluye conductas apropiadas con los residuos y el cuidado de parques y jardines) y Campista (CAM, que incluye conductas de protección del ambiente durante actividades de camping y ocio).

Por lo tanto, además de tener en cuenta el desarrollo de competencias profesionales específicas relacionadas con la educación ambiental o educación para el desarrollo sostenible (Nielsen et al., 2012; Torquati, Cutler, Gilkerson $\&$ Sarver, 2013), es preciso que los educadores incorporen también en la planificación de contenidos y actividades aspectos psicosociales subyacentes a estos temas. Los resultados sugieren que se deben planificar los contenidos en educación ambiental considerando identidad personal y social que encierran la conectividad con la naturaleza y las creencias subyacentes a las preocupaciones ambientales. Además se deben tener en cuenta los tipos de conducta y la cosmovisión asociada para la promoción de conductas de conservación, como por ejemplo en términos de ideología política y religiosidad.

En relación con el segundo objetivo, se encuentra que la conectividad con la naturaleza aumenta después de una experiencia de contacto directo con un ambiente natural, en el contexto de una actividad de educación ambiental. En un principio se esperaría que una imagen de sí mismo fuese relativamente estable, y su relación le aportara parte de esa estabilidad a la conectividad con la naturaleza. Aunque los resultados del primer estudio van en este sentido, pues no se observan variaciones en conectividad con la naturaleza según variables sociodemográficas ni socioculturales, hay suficiente evidencia acerca de la sensibilidad contextual del self (e.g. Kashima et al., 2004), del self metapersonal relacionado con la conectividad (e.g. Arnocky et al., 2007), y de los cambios en la misma conectividad después de una experiencia de contacto directo con la naturaleza (e.g. Mayer et al., 2009; Schultz \& Tabanico, 2007), como también se ha observado en el estudio dos del presente trabajo. En este sentido pierde consistencia la hipótesis biofílica de la conectividad, sobre todo al no haber información concluyente de otras investigaciones acerca de su carácter implícito (Olivos \& Aragonés, 2013a).

Por otra parte, como se ha detallado al analizar los resultados cualitativos del estudio, el itinerario hace emerger una serie de afectos relacionados con la naturaleza. Se observan reiteradas expresiones del paisaje percibido a lo largo del recorrido del que se deducen algunas propiedades colativas, tales como la novedad, al mencionarse contrastes del tipo ciudad-montaña, urbanización-bosque, ruidosonidos, o la complejidad, al nombrarse el ecosistema de montańa, o la comunidad de robles. Predominan también en el relato las emociones relacionadas con el agrado o bienestar.

Se repiten patrones de contenido similares en las descripciones de las experiencias educativas de Tomashow (1995) con participantes del Asia tropical, la sabana Africana, ciudades Latinoamericanas, valles de Europa o granjas Norteamericanas. El autor señala:

\footnotetext{
ellos tienen recuerdos de lugares especiales de su infancia, formados a través de sus conexiones con la tierra vía algún tipo de experiencia emocional, base de sus lazos con la tierra o sus entornos [...] recuerdos de experiencias de juego, exploración, descubrimiento, aventura, incluso peligro, imaginación, e independencia (p.9).
}

Los contenidos registrados en la presente investigación corresponden a elementos muy específicos relacionados a recuerdos y experiencias de contacto con la naturaleza que sucedieron en la temprana infancia o adolescencia, similar a los obtenidos por Schroeder (2007) y Tomashow (1995). Dichos eventos tuvieron lugar de la mano de algún familiar significativo o grupo de amigos relevantes. Diversas investigaciones han aportado evidencia en este sentido, de cómo las experiencias tempranas con la naturaleza en la infancia y la juventud desempeñan un papel importante (e.g. Chawla \& Cushing, 2007; Davis, Rea \& Waite, 2006; Wells \& Lekies, 2006). 
Los resultados del estudio dos en su conjunto también pueden ser relevantes para la planificación de actividades en educación ambiental. Las acciones individuales tienen un impacto sobre el medio ambiente, cuya conciencia moviliza ciertos tipos de preocupaciones ambientales que pueden contribuir a ampliar la imagen de sí mismo que encierra la Conectividad con la Naturaleza más allá de los límites del Yo y de las obligaciones sociales condicionadas por un modelo de sociedad antropocéntrico. Estas actividades no solo tienen efecto en la conservación del entorno, sino también en el desarrollo de identidades positivas y duraderas, sobre todo cuando se llevan a cabo en la temprana infancia y adolescencia.

A pesar de su utilidad para la planificación de contenidos y actividades en educación ambiental, los resultados de este trabajo deben interpretarse con cautela. Ello debido principalmente a las limitaciones del presente estudio, que no cuenta con un grupo de control claramente definido, aunque tiene una línea base comparación para la medida longitudinal. Del mismo modo, carece de un procedimiento de análisis que permita integrar los resultados cuantitativos y cualitativos en un mismo protocolo. En este sentido se está llevando a cabo una nueva experiencia que, además de superar estas limitaciones metodológicas, está ampliando la muestra a otros contextos para avanzar en la validez transcultural de sus resultados.

En suma, la conectividad con la naturaleza corresponde a un tipo de identidad ambiental, probablemente provenientes tanto de las sensaciones presentes de agrado como de las experiencias pasadas con entornos naturales en la infancia o adolescencia. La relación entre las creencias egobiocéntricas y la conectividad, sugiere que la relación de pertenencia con la naturaleza está centrada en las sensaciones de satisfacción personal, al estilo de los efectos restauradores de los ambientes naturales, como se ha mencionado anteriormente. Esta es una línea de investigación que puede contribuir a explorar la disposición favorable hacia el medio ambiente, y los efectos positivos de interactuar con ellos, en áreas protegidas u otro tipo de entorno con naturaleza. Estos temas constituyen oportunidades para el estudio de la conectividad, así como de sus alcances prácticos en el campo de la promoción de salud y de la educación ambiental.

\section{Referencias}

Amérigo, M., Aragonés, J.I., De Frutos, B., Sevillano, V., \& Cortés, B. (2007). Underlying Dimensions of Ecocentric and Antropocentric Environmental Belief. The Spanish Journal of Psychology, 10, 97-103.

Arnocky, S., Stroink, M., \& DeCicco, T. (2007). Self-construal Predicts Environmental Concern, Cooperation, and Conservation. Journal of Environmental Psychology, 27, 255-264. doi: 10.1016/j.jenvp.2007.06.005.

Brügger, A., Kaiser, F.G., \& Roczen, N. (2011). One for All? Connectedness to Nature, Inclusion of Nature, Environmental Identity, and Implicit Association with Nature. European Psychologist, 16, 324-333. doi: 10.1027/1016-9040/a000032

Chawla, L. \& Cushing, D. (2007). Education for Strategic Environmental Behavior. Environmental Education Research. 13, 437-452.: doi: 10.1080/13504620701581539

Corraliza, J.A. (2000). Emoción y Ambiente. En J.I. Aragonés y M. Amerigo (Coord.), Psicología Ambiental (pp. 59-76). Madrid: Pirámide.

Davis, B., Rea, T., \& Waite, S. (2006). The Special Nature of Outdoors: Its Contribution to the Education of Children at Ages 3-11. Australian Journal of Outdoor Education. 10, 3-12.

Erdoğan, M. (2011). The Effects of Ecology-Based Summer Nature Education Program on Primary School Students' Environmental Knowledge, Environmental Affect and Responsible Environmental Behavior. Educational Sciences: Theory \& Practice, 11, 2233-2237.

Ernst, J., \& Theimer, S. (2011). Evaluating the Effects of Environmental Education Programming on Connectedness to Nature. Environmental Education Research, 17, 577-598. doi: 10.1080/13504622.2011.565119.

Fangler, J., \& Schwarzer, C. (July, 2008). Is There a Self-concept Change After a Participation in Outdoor Education Programs?. An Evaluation Study. Communication presented at XXIX International Congress of Psychology. Berlin, Germany.

González-Gaudiano, E. (2005). Education for Sustainable Development: configuration and meaning. Policy Futures in Education, 3, 243-250. doi: 10.2304/ piie.2005.3.3.2 
Hagerhall, C.M. (2001). Consensus in Landscape Preference Judgments. Journal of Environmental Psychology, 21, 83-92. doi: 10.1006/jevp.2000.0186.

Han, K.T. (2007). Responses to Six Major Terrestrial Biomes in Terms of Scenic Beauty, Preference, and Restorativeness. Environment and Behavior, 39, 529-556. doi: 10.1177/0013916506292016.

Hesselink, F., van Kempen, P.P., \& Wals, A. (2000). International Debate on Education for Sustainable Development. IUCN. Gland y Cambridge.

Hoot, R.E., \& Friedman, H. (2011). Connectedness and Environmental Behavior: Sense of Interconnectedness and Pro-Environmental Behavior. International Journal of Transpersonal Studies, 30, 89-100.

Kaplan, R. (2001). The Nature of the View From Home Psychological Benefits. Environment and Behavior, 33, 507-542. doi: 10.1177/00139160121973115.

Kaplan, S. (1995). The Restorative Benefits of Nature: Toward an Integrative Framework. Journal of Environmental Psychology, 15, 169-182. doi: 10.1016/0272-4944(95)90001-2.

Kashima, Y., Kashima, E., Farsides, T., Kim, U., Strack, F., Werth, L., \& Yuki, M. (2004). Culture and context-sensitive self: The amount and meaning of context-sensitivity of phenomenal self differ across cultures. Self and Identity, 3, 125-141. doi: 10.1080/13576500342000095.

Liefländer, A.K., Fröhlich, G., Bogner, F.X., \& Schultz, P.W. (2013). Promoting connectedness with nature through environmental education. Environmental Education Research, 19, 370-384. doi: http://dx.doi. org/:10.1080/13504622.2012.697545.

Martens, D., \& Bauer, N. (July, 2008). Do presentation models of nature influence the effect on human wellbeing?. A comparison of laboratory and field results. Communication presented at XXIX International Congress of Psychology. Berlin, Germany.

Mayer, F.S., \& Frantz, C.M. (2004). The connectedness to nature scale: a measure of individuals' feeling in community with nature. Journal of Environmental Psychology, 24, 503-515. doi: 10.1016/j. jenvp.2004.10.001.

Mayer, F.S., Frantz, C.M., Bruehlman-Senecal, E., \& Dolliver, K. (2009). Why Is Nature Beneficial? The Role of
Connectedness to Nature. Environment and Behavior, 41, 607-643. doi: 10.1177/0013916508319745.

Navarro, O. (2011) Les enjeux socio-environnementaux du développement durable en Amérique du Sud. Considérations à partir du cas colombien, Développement durable et territoires, 2(3). doi: 10.4000/developpementdurable.9045.

Nielsen, W., Andersen, P., Hurley, A., Sabljak, V., Petereit, A.L, Hoskin, V., \& Hoban, G. (2012). Preparing Action Competent Environmental Educators: How Hard Could It Be?. Australian Journal of EnvironmentalEducation, 28, 92-107. doi: 10.1017/ aee.2013.3.

O'Donoghue, R. (2006). Locating the Environmental in Environmental Education Research: A Review of Research on Nature's Nature, its Inscription in Language and Recent Memory Work on Relating to the natural world. Environmental Education Research, 12, 345-357. doi: 10.1080/13504620600799117

Olivos, P. (2006). Oportunidades para una educación ambiental para el desarrollo sustentable, desde una experiencia profesional en Chile. En J.A. Corraliza, J. Berenguer, y R. Martín. (Eds), Medio Ambiente, Bienestar Humano y Responsabilidad Ecológica (pp. 194-197). La Laguna: Ed. Resma y Fundación General de la UAM.

Olivos, P., \& Aragonés, J.I. (2011). Psychometric Properties of the Environmental Identity Scale (EID). Psyecology, 2, 65-74. doi: 0.1174/217119711794394653.

Olivos, P. Aragonés, J.I., \& Amérigo, M. (2011). The Connectedness with Nature Scale and its Relationship with Environmental Beliefs and Identity. Internacional Journal of Hispanic Psychology, 4, 5-19.

Olivos, P., \& Aragonés, J.I. (2013a). Test de Asociaciones Implícitas con la Naturaleza: aplicación en España del IAT-Nature. Revista de Psicología Social, 28, 237-245. doi: 10.1174/021347413806196672.

Olivos. P., \& Aragonés, J.I. (2013b). Medio ambiente, self y conectividad con la naturaleza. Revista Mexicana de Psicología. En prensa.

Olivos, P., \& González, R. (2005). Análisis de un concepto local de educación para el desarrollo sustentable. Revista Ideas Ambientales, 2, 6-16.

Olivos, P., \& Moyano, E. (2004). Proposiciones desde la educación ambiental para la prevención y la 
descontaminación ambiental. En H. Günther, J.Q. Pinheiro, y R.S. Lobo. (Eds.), Psicologia Ambiental, entendendo as relaçóes do homem com seu ambiente (pp. 101-113). Brasil: Alínea.

Olivos. P., \& Talayero, F. (Julio, 2013). Conectividad con la Naturaleza e Identidad Ambiental como predictores del Comportamiento y Compromiso Proambientales. Comunicación presentada en el XXXIV Congreso Interamericano de Psicología. Brasilia, Brasil.

Perrin, J.L., \& Benassi, V.A. (2009). The Connectedness to Nature Scale: A Measure of Emotional Connection to Nature?. Journal of Environmental Psychology, 29, 434-440. doi: 10.1016/j.jenvp.2009.03.003.

Schroeder, H.W. (1991). Preference and Meaning of Arboretum Landscapes: Combining quantitative and qualitative data. Journal of Environmental Psychology, 11,231-248. doi: 10.1016/S0272-4944(05)80185-9.

Schroeder, H.W. (2002). Experiencing Nature in Special Places. Journal of Forestry, 100, 8-14.

Schroeder, H.W. (2007). Place Experience, Gestalt, and the human-nature relationship. Journal of Environmental Psychology, 27, 293-309. doi: 10.1016/j.jenvp.2007.07.001.

Schultz, P., Shriver, C., Tabanico, J., \& Khazian, A. (2004). Implicit connections with nature. Journal of Environmental Psychology, 24, 31-42. doi: 10.1016/ S0272-4944(03)00022-7.

Schultz, P., \& Tabanico, J. (2007). Self, Identity, and the Natural Environment: Exploring Implicit
Connections with Nature. Journal of Applied Social Psychology, 37, 1219-1247. doi: 10.1111/j.15591816.2007.00210.x

Sevillano, V. (2007). Empatía y cognición social en la preocupación por el medio ambiente (Tesis doctoral). Facultad de Psicología, Universidad Complutense de Madrid, Madrid, España.

Staats, H., Gatersleben, B., \& Hartig, T. (1997). Change in Mood as a Function of Environmental Design: Arousal and Pleasure on a Simulated Forest Hike. Journal of Environmental Psychology, 17, 283-300. doi: 10.1006/jevp.1997.0069.

Strauss, A., \& Corbin, J. (1994). Grounded Theory Methodology. In N.K. Denzin \& Y.S. Lincoln (Eds.), Handbook of Qualitative Research (pp. 217-285). Thousand Oaks: Sage Publications.

Thomashow, M. (1995). Ecological Identity, becoming a reflective environmentalist. London: MIT Press.

Torquati, J., Cutler, K., Gilkerson, D., \& Sarver, S. (2013). Early Childhood Educators' Perceptions of Nature, Science, and Environmental Education. Early Education and Development, 24, 721-743. doi: 10.1080/10409289.2012.725383

Wells, N., \& Lekies, K. (2006). Nature and the life course: Pathways from childhood nature experiences to adult environmentalism. Children, Youth and Environments. $16,1-24$. 
